\title{
HARTMAGNETISCHE WERKSTOFFE
}

Die Herausforderung bestand darin, Prüfverfahren zu entwickeln, die die realen Belastungen von $\mathrm{NdFeB}$-Sintermagneten in Elektromotoren zeitgerafft abbilden und eine zuverlässige Beurteilung der Durabilität eines solchen Werkstoffs zulassen. Für die Anwendung im Elektromotoren ist es insbesondere erforderlich, elektrische, magnetische und korrosive Eigenschaften von NdFeB-Sintermagneten im Zusammenhang und nicht (wie in den bisher etablierten Prüfverfahren) isoliert voneinander zu betrachten. Dies umfasst die Korrosion der Werkstoffe durch den Kontakt

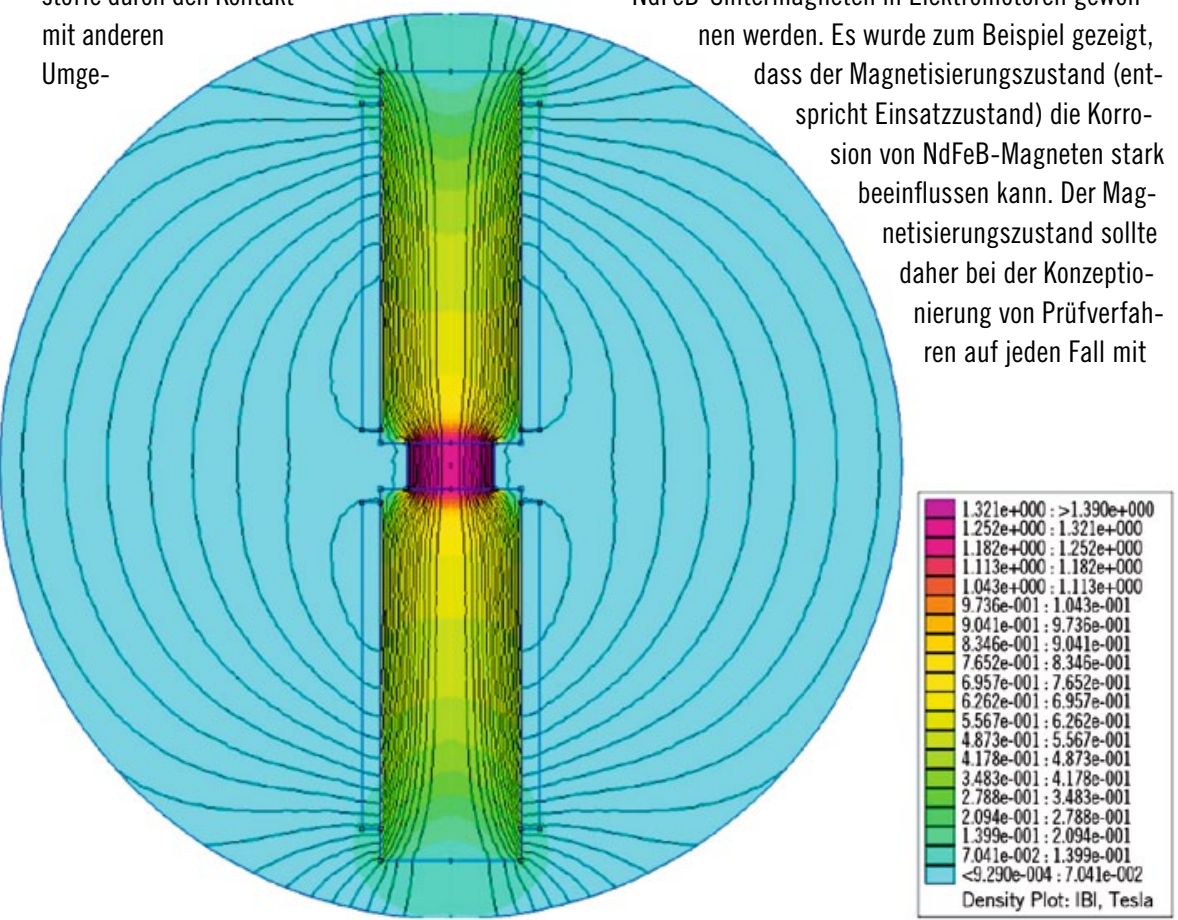

Verteilung der Magnetflussdichte in der Magnetprobe und den Eisenkernen

\section{VERDUNSTUNGSEMISSIONEN IM LUFTANSAUGSYSTEM VON OTTOMOTOREN}

Im Vorgängerprojekt „Verdunstungsemissionen I“ wurde für Simulationen am Laborrohrmodell und an vereinfachten Bauteilgeometrien bereits gezeigt, dass durch eine adaptive Zeitschrittweitensteuerung (das Programm wählt in jedem Zeitschritt eine neue angepasste Schrittweite, um eine vorgegebene Genauigkeit möglichst effizient zu erreichen) und durch die Skalierung der Diffusionskoeffizienten (der Diffusionskoeffizient wird um mehrere GröBenordnungen erhöht, wodurch eine umgekehrte Skalierung der Zeitachse erreicht wird) deutliche Reduzierungen der Rechenzeiten und der Hardwarekapazitäten erzielt werden können. Die Skalierung der Diffusionskoeffizienten reduziert die bungsmedien, ihr Verhalten in magnetischen Wechselfeldern sowie die direkte Korrelation dieser Ergebnisse mit den magnetischen Kenndaten.

Im Rahmen des Projekts wurden Korrosionstestverfahren für kommerzielle NdFe-B-Sintermagnete mit verschiedenen Beschichtungen entwickelt. Unter anderem wurden die Magnete systematisch in Getriebeöl anwendungsrelevanten Temperaturzyklen ausgesetzt und anschließend magnetisch sowie strukturell charakterisiert. Auf diese Weise konnten relevante Erkenntnisse für den Einsatz von NdFeB-Sintermagneten in Elektromotoren gewonwerden. Es wurde zum Beispiel gezeigt, spricht Einsatzzustand) die Korrosion von NdFeB-Magneten stark netisierungszustand sollte daher bei der KonzeptioPrüfverfahren auf jeden Fall mit

berücksichtigt werden. Die entwickelten Testverfahren geben dem Anwender die Möglichkeit, die Durabilität von NdFeB-Sintermagneten unter Einsatzbedingungen besser zu beurteilen sowie Werkstoffe hoher und niedriger Materialgüte voneinander zu unterscheiden. Das Vorhaben wurde im Rahmen des $\mathrm{CO}_{2}$-Sonderforschungsprogramms der Forschungsvereinigungen Antriebstechnik (FVA) und Verbrennungskraftmaschinen (FVV) durch Industriemittel finanziert.

\section{FORSCHUNGSSTELLE: \\ LEIBNIZ INSTITUT FÜR \\ FESTKÖRPER- UND WERKSTOFF- \\ FORSCHUNG, DRESDEN \\ OBMANN:}

DR.-ING. FLORIAN LAMPMANN, DAIMLER AG

\section{FORSCHUNGSVEREINIGUNG VERBRENNUNGSKRAFT- MASCHINEN E.V.}

Die FVV wurde 1956 gegründet und hat sich zum weltweit einmaligen Netzwerk der Motoren- und Turbomaschinenforschung entwickelt. Sie treibt die gemeinsame, vorwettbewerbliche Forschung in der Branche voran und bringt Industrieexperten und Wissenschaftler an einen Tisch, um die Wirkungsgrade und Emissionswerte von Motoren und Turbinen kontinuierlich zu verbessern zum Vorteil von Wirtschaft, Umwelt und Gesellschaft. Außerdem fördert sie den wissenschaftlichen Nachwuchs. Mitglieder sind kleine, mittlere und große Unternehmen der Branche: Automobilunternehmen, Motoren- und Turbinenhersteller sowie deren

Nutzung mit dem Ziel, eine Rechenzeit von weniger als $10 \mathrm{~h}$ zu erreichen, musste das Berechnungsverfahren bezüglich der zu wählenden Eingabeparameter methodisch weiterentwickelt, validiert und optimiert werden. Das Vorhaben wurde aus Eigenmitteln der FVV finanziert.

FORSCHUNGSSTELLE: INSTITUT FÜR CHEMISCHE VERFAHRENSTECHNIK, UNI STUTTGART OBMANN:

DR.-ING. FRANK PFEIFFER, MANN+HUMMEL GMBH

\section{Zulieferer.}

Kontakt:

Dipl.-Ing. Stefanie Jost-Köstering

Presse- und Öffentlichkeitsarbeit

Forschungsvereinigung

Verbrennungskraftmaschinen e.V.

Lyoner Straße 18। 60528 Frankfurt/Main

Telefon +4969 6603-1531

Fax +49 69 6603-2531

E-Mail sjk@fvv-net.de

http://www.fvv-net.de

FVV 